\title{
Non-Classical Progenitor Mononuclears in Metabolic Syndrome: The Role of Serum 25-Hydroxyvitamin D3
}

\section{Berezin $\mathrm{AE}^{1 *}$, Kremzer $\mathrm{AA}^{2}$, Martovitskaya $\mathrm{YV}^{3}$ and Berezina $\mathrm{TA}^{4}$}

${ }^{1}$ Department of Internal Medicine, State Medical University, 26, Mayakovsky av., Zaporozhye, Ukraine

${ }^{2}$ Department of Clinical Pharmacology, State Medical University, Zaporozhye, Ukraine

${ }^{3}$ Clinical Laboratory "Dia-Service", Department of Clinical Immunology, Zaporozhye, Ukraine

${ }^{4}$ Private center "Vita-Center", Zaporozhye, Ukraine

\begin{abstract}
Background: There is evidence pivotal role of vitamin $D$ in pathogenesis of metabolic syndrome (MetS) affected worsening endogenous repair system.

Objective: This study was conducted to investigate the pattern of circulating progenitor mononuclears in MetS patients with low 25(OH)D3 levels.

Methods: The study prospectively evolved 47 patients with MetS and 35 healthy volunteers. Circulating level of $25(\mathrm{OH}) \mathrm{D} 3$ and other biomarkers were measured at baseline of the study. Mononuclear progenitor cells were determined using the flow cytometric technique.

Results: Metabolic syndrome (MetS) patients from entire group were divided in to four cohorts depending on $25(\mathrm{OH})$ D3 level $>100 \mathrm{nmol} / \mathrm{L}(\mathrm{n}=10), 50$ to $100 \mathrm{nmol} / \mathrm{L}(\mathrm{n}=12) ; 30$ to $50 \mathrm{nmol} / \mathrm{L}(\mathrm{n}=14)$, and $<30 \mathrm{nmol} / \mathrm{L}(\mathrm{n}=11)$. There were sufficiently distinguishes between cohorts patients with Metabolic syndrome (MetS) in HbA1c $(P=0.038)$, HOMAIR $(P=0.042)$, triglycerides $(P=0.044)$, osteoprotegerin $(P=0.028)$, adiponectin $(P=0.018)$, HDL-C $(P=0.036)$, and $\mathrm{CD} 14^{+} \mathrm{CD} 309^{+} \mathrm{Tie}-2^{+}$cells. Vitamin $\mathrm{D}$ deficiency status in multivariate log-regression model appeared to be remained an independent predictor to depletion of numerous of $\mathrm{CD} 14^{+} \mathrm{CD} 309^{+}$Tie- $2^{+}$cells $(\mathrm{OR} 1.12 ; 95 \% \mathrm{Cl} 1.06$ to $1.19 ; \mathrm{P}=0.002$ ), whereas other vitamin $\mathrm{D}$ statuses were not found as predictors. Osteoprotegerin, hs-CRP, adiponectin have exhibited an independent impact on depletion of numerous of CD $14^{+} \mathrm{CD} 309^{+} \mathrm{Tie}-2^{+}$cells.

Using C-statistics we found that three biomarkers (osteoprotegerin, hs-CRP, and adiponectin) avoid to improve significantly predictive model based on plasma level of $25(\mathrm{OH}) \mathrm{D} 3<30 \mathrm{nmol} / \mathrm{L}$ for decreased numerous of $\mathrm{CD} 14^{+}$ CD309+Tie- $2^{+}$cells. In patient study population for category-free NRI, $3 \%$ of events $(p=0.16)$ and $4 \%$ of non-events $(p=0.12)$ were correctly reclassified by the addition of circulating inflammatory biomarkers (hs-CRP, osteoprotegerin and adiponectin) to the base model for decreased numerous of CD14+ CD309+Tie-2+ cells.
\end{abstract}

Conclusion: In conclusion, we found that vitamin D status especially low level of 25(OH)D3 may associate with depletion of circulating number of proangiogenic progenitor mononuclears in MetS patients.

Keywords: Metabolic syndrome; Vitamin D; Cardiovascular risk factors; Progenitor mononuclears; Inflammation

Abbreviations: ANOVA: Analysis of Variance; AUC: Area Under Curve; BMI: Body Mass Index; CV: Cardio Vascular; EPCs: Endothelial Progenitor Cells; GFR: Glomerular Filtration Rate; hs-CRP: High Sensitive C-Reactive Protein; HDL-C: High-Density Lipoprotein Cholesterol; IQR: Interquartile Range; IDI: Integrated Discrimination Indices; LDL-C: Low-Density Lipoprotein Cholesterol; MetS: Metabolic Syndrome; NRI: Net-Reclassification Improvement; OPG: Osteoprotegerin; T2DM: Type 2 Diabetes Mellitus; TG: Triglycerides; BP: Blood Pressure; sRANKL: Serum Receptor Activator of NF- $\kappa B$ ligand; HbAlc: Glycated Hemoglobin; CI: Confidence Interval.

\section{Introduction}

Metabolic syndrome (MetS) is a cluster of cardiovascular (CV) risk factors, including central obesity, glucose homeostasis, insulin resistance, hypertension and atherogenic dyslipidemia, appears to have an increased prevalence worldwide [1]. Recent clinical and observation trials have shown that the patients with MetS exhibit near 2-fold increased risk of $\mathrm{CV}$ disease and events, asymptomatic atherosclerosis, all cause and CV death rate $[2,3]$.

There are evidence regarding the pivotal role of vitamin $\mathrm{D}$ in pathogenesis of CV disease, diabetes mellitus, obesity, and MetS [4]. The reliable indicator of body vitamin D status is serum concentration of 25-hydroxyvitamin D [25(OH)D3]. Low serum 25(OH)D3 levels have been directly linked to MetS, whereas the overall risk of MetS in general population was not probably associated with $25(\mathrm{OH}) \mathrm{D} 3$ concentration [5-7]. However, vitamin D deficiency defined as serum 25(OH)D3 level $<20 \mathrm{ng} / \mathrm{mL}$ is associated with insulin resistance (IR), decreased insulin secretion by pancreatic beta-cells, inflammation intensity, lower circulating adiponectin, and activation of the renin-angiotensin system [8,9]. Moreover, abdominal obesity, hypertension, endothelial dysfunction and atherogenic dyslipidemia have exhibited a closely association with low level of circulating 25(OH)D3 [10,11]. 25(OH)D3 may act through vitamin D receptors (VDR), which mediate calcium efflux into target cells (beta-cells, cardiomyocytes, pre-maturated

*Corresponding author: Alexander Berezin E, Department of Internal Medicine State Medical University of Zaporozhye, 26, Mayakovski av., Zaporozhye, UA-69035, Ukraine, Tel: +380612894585; E-mail: dr_berezin@mail.ru or aeberezin@gmail.com

Received April 21, 2016; Accepted May 06, 2016; Published May 13, 2016

Citation: Berezin AE, Kremzer AA, Martovitskaya YV, Berezina TA (2016) Non-Classical Progenitor Mononuclears in Metabolic Syndrome: The Role of Serum 25-Hydroxyvitamin D3. Clin Med Biochem Open Access 2: 115. doi:10.4172/2471-2663.1000115

Copyright: (c) 2016 Berezin AE, et al. This is an open-access article distributed under the terms of the Creative Commons Attribution License, which permits unrestricted use, distribution, and reproduction in any medium, provided the original author and source are credited. 
adipocytes, mononuclear progenitor cells liver cells, and skeletal muscle cells) and attenuates secretion of inflammatory cytokines via direct stimulation of transcription factor nuclear factor- $\kappa \mathrm{B}(\mathrm{NF}-\kappa \mathrm{B})$ [12]. Overall, VDR and the $1 \alpha$-hydroxylase enzyme, which catalyzes the conversion of 25(OH)D3 to 1,25-dihydroxyvitamin D (1,25(OH)2D) and expresses on surface of several cell types, may contribute to multiple metabolic regulation of target cells including stem cells and progenitor cells [13]. Finally, the oxidative stress, inflammatory cytokines, and vitamin D status via epigenetic mechanisms may alter functionality of progenitor endothelial and mononuclear cells that are essential for endothelium reparation, neovascularization and maintenance of endothelial integrity [14].

The role of recruiting and differentiation of progenitor mononuclear cells that are involved in the pathophysiology of MetS remains controversial $[15,16]$. Indeed, dysfunction of progenitor mononuclear cells may play a prominent role in worsening of endothelium reparation due to direct endothelium damage, endothelial dysfunction, microvascular inflammation, and oxidative stress [17]. Contrary, endothelial function might be impaired due to altered maturation / commitment of progenitor mononuclears, rather than a simple decrease in their production in the bone marrow and that this process might be under control of 25(OH)D3 [18].

Numerous studies confirmed the relationship between $25(\mathrm{OH}) \mathrm{D} 3$ and depletion of circulating endothelial progenitor cells in diabetes mellitus $[19,20]$. However, the relationships between the number of circulating progenitor mononuclear cells in MetS patients and 25(OH) D3 level is not fully clear. The objective of the study: to investigate the pattern of circulating progenitor mononuclears in MetS patients with low 25(OH)D3 levels.

\section{Methods}

\section{Study design and patients' study population}

Forty seven patients with MetS and 35 healthy volunteers were included in the study. The enrolment has done within winter months during three consequents years to minimize an effect of other seasons. We have included MetS patients without known type 2 diabetes mellitus (T2DM) and known coronary artery disease. The National Cholesterol Education Program Adult Treatment Panel III criteria [21] to establish MetS was used. All subjects have given written informed consent. The study has performed with the Declaration of Helsinki.

\section{Anthropometric measurements}

Anthropometric measurements including height (to the nearest centimeter) and body weight (to the nearest of a kilogram) were made using standard procedures [22] after an overnight fast at the first visit. The waist circumference was measured from the front at the narrowest point between the rib cage and iliac crest after full expiration.

Systolic and diastolic blood pressure was measured using an automatic blood pressure monitor (Omron, Japan). Three readings were taken and the average was used in the analysis.

\section{Current smoking status}

Current smoking was defined as consumption of one cigarette daily for three months.

\section{Treatment and concomitant medications}

Subjects with MetS were treated with life-style modification and diet, therefore metformin has given in 12 patients.

\section{Calculation of glomerular filtration rate}

Glomerular filtration rate (GFR) was calculated with CKD-EPI formula [23].

\section{Insulin resistance determination}

Insulin resistance was determined by the homeostasis model assessment (HOMA-IR) [24] using the following formula:

HOMA-IR $(\mathrm{mmol} / \mathrm{L} \times \mu \mathrm{U} / \mathrm{mL})=$ fasting glucose $(\mathrm{mmol} / \mathrm{L}) \times$ fasting insulin $(\mu \mathrm{U} / \mathrm{mL}) / 22.5$

Insulin resistance was defined when estimated HOMA-IR value was over $2.77 \mathrm{mmol} / \mathrm{L} \times \mu \mathrm{U} / \mathrm{mL}$ as it was defined previously [25].

\section{Measurement of circulating biomarkers}

All measurements were performed at baseline at 7-8 a.m. Blood samples were collected into cooled silicone test tubes with $2 \mathrm{~mL}$ of $5 \%$ Trilon B solution. The centrifugation of blood samples were done at $6,000 \mathrm{rpm}$ for 3 minutes. Plasma was refrigerated immediately and then stored at a temperature $-70^{\circ} \mathrm{C}$.

Measurements of serum C-reactive protein (hs-CRP), RANKL, osteoprotegerin (OPG), and adiponectin have used high-sensitive ELISA kits (R\&D Systems GmbH, Wiesbaden-Nordenstadt, Germany).

Fasting insulin level was measured by a double-antibody sandwich immunoassay (Elecsys 1010 analyzer, F. Hoffmann-La Roche Diagnostics, Mannheim, Germany). The lower detection limit of insulin level was $1.39 \mathrm{pmol} / \mathrm{L}$.

Concentrations of total cholesterol (TC), cholesterol of low-density lipoproteins (LDL-C), and cholesterol of high-density lipoproteins (HDL-C) were measured by enzymatic colorimetric method according standardized methodology on Beckman Synchron LX20 chemistry analyzer.

\section{Measurement of plasma 25-hydroxy vitamin D (25(OH)D3)}

Circulating level of 25(OH)D3 was measured using an ELISA kit (BG Medicine, Germany). The plasma 25(OH)D3 levels > $100 \mathrm{nmol} / \mathrm{L}$ are defined as optimal vitamin D status and levels from 50 to $100 \mathrm{nmol} / \mathrm{L}$ are defined as adequate. Serum levels of $25(\mathrm{OH}) \mathrm{D}<50 \mathrm{nmol} / \mathrm{L}$ are proposed to define inadequate vitamin $\mathrm{D}$ status, and values $<30 \mathrm{nmol} / \mathrm{L}$ represent vitamin $\mathrm{D}$ deficiency.

\section{Assay of circulating mononuclear progenitor cell subsets}

The flow cytometric technique (FCT) was used for measurement of circulating cell numbers using High-Definition Fluorescence Activated Cell Sorter (HD-FACS) methodology [26]. The cell subsets were phenotyped using forward scatter characteristic (FSC) and side scatter characteristic (SSC) profiles accordingly standard protocol [27]. Expression of cell surface proteins, i.e., anti-CD45 FITS (BD Biosciences, San Jose, CA, USA), anti-CD34 FITS (BD Biosciences), anti-VEGFR-2 known as anti-CD309 (BD Biosciences), anti-Tie2 (BD Biosciences) and anti-CD14 (BD Biosciences) were stained for cells identification. Erythrocytes were eliminated using UTILIZE wash solution after lysis and fixed. After then the samples were centrifuged at $200 \times \mathrm{g}$ for $15 \mathrm{~min}$ and analyzed. Double- and triple-positive events were determined using Boolean principles. We used the fluorescence minus one technique to provide control for measurements.

\section{Identification of mononuclear progenitors}

Mononuclear progenitors were defined as CD45-/CD34+/ CD309+(VEGFR2+) cells. For each tube we analyzed 500,000 events 
using quadrant methods to determine number of mononuclear progenitors. The FITC-labeled isotype control has implemented the same gate and window settings. Angiogenic mononuclear progenitor phenotype for was defined as $\mathrm{CD} 14^{+} \mathrm{CD} 309^{+}$, CD $14^{+} \mathrm{Tie}^{2} 2^{+}$and $\mathrm{CD}_{309^{+}} \mathrm{Tie}^{-} 2^{+}$antigen presentation. The reproducibility of cell subsets' measurements using the standard protocol was 3.5\%.

\section{Statistical analysis}

Statistical analysis of the results obtained was performed in SPSS system for Windows, Version 23 (SPSS Inc, Chicago, IL, USA). The data were presented as mean $(M)$ and standard deviation $( \pm S D)$ or 95\% confidence interval (CI); as well as median (Me) and 25\%-75\% interquartile range (IQR). To compare the main parameters of patient cohorts, two-tailed Student t-test or Shapiro-Wilk U-test were used. To compare categorical variables between groups, Chi2 test $\left(\chi^{2}\right)$ and Fisher $F$ exact test were used. Predictors of depleted progenitor mononuclears in MetS patients were examined in univariate and multivariate logregression analysis. C-statistics, integrated discrimination indices (IDI) and net-reclassification improvement (NRI) were utilized for prediction performance analyses. A two-tailed probability value of $<0.05$ was considered as significant.

\section{Results}

The age and sex proportion regarding patients with MetS and healthy volunteers were similar (Table 1). There were significant differences between healthy subjects and MetS in antropomentics (BMI, waist circumference), $\mathrm{CV}$ factors and Framingham risk score. In contrast, fasting serum glucose, $\mathrm{HbAlc}$, insulin, inflammatory biomarkers (hsCRP, osteoprotegerin, sRANKL, and adiponectin) were higher in patients with MetS compared with controls. Nevertheless, circulating 25(OH)D3 level in MetS patients was lower than in healthy volunteers.

Number of circulating mononuclear progenitor cells labeled $\mathrm{CD} 45^{-\mathrm{CD}} 34^{+}, \mathrm{CD}_{14} 4^{+} \mathrm{CD} 309^{+}, \mathrm{CD} 14^{+} \mathrm{Tie}_{-} 2^{+}, \mathrm{CD} 309^{+} \mathrm{Tie}^{-2^{+}}$and $\mathrm{CD} 14^{+} \mathrm{CD} 309^{+} \mathrm{Tie}^{+}{ }^{+}$were significantly higher in MetS patients compared with healthy volunteers. Figure 1 is shown the sample of flow cytometry picture represented $\mathrm{CD} 14^{+} \mathrm{CD} 309+$ anti-Tie2 ${ }^{+}$cells in MetS patients depending on the plasma level of 25(OH)D3.

MetS patients from entire group were divided in to four cohorts depending on 25(OH)D3 level $>100 \mathrm{nmol} / \mathrm{L}(\mathrm{n}=10), 50$ to $100 \mathrm{nmol} / \mathrm{L}$ $(\mathrm{n}=12) ; 30$ to $50 \mathrm{nmol} / \mathrm{L}(\mathrm{n}=14)$, and $<30 \mathrm{nmol} / \mathrm{L}(\mathrm{n}=11)$ defined as adequate, inadequate, insufficiency and deficiency vitamin $\mathrm{D}$. The mean plasma levels of 25(OH)D3 in each MetS cohorts are presented in the Figure 2.

Table 2 is summarized characteristics regarding MetS depending vitamin D status defined accordingly plasma level of 25(OH)D3. One can see, patients with deficiency of vitamin $\mathrm{D}$ appear to be haven higher age $(\mathrm{P}=0.046)$ and frequently defined dyslipidemia $(\mathrm{P}=0.044)$. Therefore, there were sufficiently distinguishes between cohorts patients with MetS in HbA1c $(\mathrm{P}=0.038)$, HOMA-IR $(\mathrm{P}=0.042)$, triglycerides $(\mathrm{P}=0.044)$, osteoprotegerin $(\mathrm{P}=0.028)$, adiponectin $(\mathrm{P}=0.018)$, HDL-C $(\mathrm{P}=0.036)$, and $\mathrm{CD} 14^{+} \mathrm{CD} 309^{+}$Tie- $2^{+}$cells.

The univariate linear regression was determined association between plasma level of 25(OH)D3, numerous of progenitor mononuclear cells, $\mathrm{CV}$ risk factors, and other biomarkers. The data have shown that plasma level of 25(OH)D3 has positively associated with numerous of $\mathrm{CD} 14^{+}$ CD309 ${ }^{+}$Tie- $2^{+}$cells $(\mathrm{r}=0.41, \mathrm{P}=0.001)$, numerous of $\mathrm{CD} 14^{+} \mathrm{Tie}-2^{+}$cells $(\mathrm{r}=0.28, \mathrm{P}=0.001)$; and inversely related with body mass index $(\mathrm{r}=-$ 0.44, $\mathrm{P}=0.001)$, osteoprotegerin $(\mathrm{r}=-0.36, \mathrm{P}=0.001)$, HbAlc $(\mathrm{r}=-0.35$, $\mathrm{P}=0.003)$, age $(\mathrm{r}=-0.35, \mathrm{P}=0.001)$, dyslipidemia $(\mathrm{r}=-0.34, \mathrm{P}=0.001)$, hs-
$\mathrm{CRP}(\mathrm{r}=-0.33, \mathrm{P}=0.001)$, triglycerides $(\mathrm{r}=-0.32, \mathrm{P}=0.001)$, Framingham risk score $(\mathrm{r}=-0.31, \mathrm{P}=0.001)$, HOMA-IR $(\mathrm{r}=0.30, \mathrm{P}=0.003)$, HDL-C $(\mathrm{r}=-0.29, \mathrm{P}=0.001)$, adiponectin $(\mathrm{r}=-0.29, \mathrm{P}=0.001)$, sRANKL $(\mathrm{r}=-0.24$, $\mathrm{P}=0.001)$, smoking $(\mathrm{r}=-0.22, \mathrm{P}=0.001)$.

Among entire group of MetS patients a closely associations between numerous of $\mathrm{CD} 14^{+} \mathrm{CD} 309^{+} \mathrm{Tie}-2^{+}$cells and osteoprotegerin $(\mathrm{r}=-0.46$, $\mathrm{P}=0.003)$, hs-CRP $(\mathrm{r}=-0.41, \mathrm{P}=0.001)$, sRANKL $(\mathrm{r}=-0.38, \mathrm{P}=0.001)$, triglycerides $(\mathrm{r}=-0.30, \mathrm{P}=0.001)$, HOMA-IR $(\mathrm{r}=0.28, \mathrm{P}=0.003)$, and adiponectin $(\mathrm{r}=-0.26, \mathrm{P}=0.001)$ was found. The numerous of $\mathrm{CD} 14^{+}{ }^{+} \mathrm{Tie}-2^{+}$cells related significantly with osteoprotegerin $(\mathrm{r}=-0.34$, $\mathrm{P}=0.003)$, hs-CRP ( $\mathrm{r}=-0.32, \mathrm{P}=0.001)$, sRANKL $(\mathrm{r}=-0.32, \mathrm{P}=0.003)$, HbA1c $(r=-0.31, P=0.001)$, and HOMA-IR $(r=0.28, P=0.001)$. No any sufficient associations between numerous of progenitor mononuclears with GFR, fasting glucose, blood pressure, and smoking were found.

\begin{tabular}{|c|c|c|c|}
\hline Variables & $\begin{array}{l}\text { Healthy } \\
\text { volunteers } \\
(n=35)\end{array}$ & $\begin{array}{l}\text { Entire cohort of } \\
\text { MetS patients } \\
\qquad(n=47)\end{array}$ & $P$ value \\
\hline Age, years & $46.12 \pm 4.22$ & $48.30 \pm 3.94$ & 0.68 \\
\hline Males, n (\%) & $23(65.7 \%)$ & $30(63.8 \%)$ & 0.86 \\
\hline BMI, $\mathrm{kg} / \mathrm{m}^{2}$ & $21.5(16.1-23.5)$ & $28.2(16.7-31.0)$ & 0.001 \\
\hline Waist circumference, sm & $78(63-89)$ & $92(69-105)$ & 0.001 \\
\hline Hypertension, n (\%) & - & $32(68.0 \%)$ & 0.001 \\
\hline Dyslipidemia, n (\%) & - & $26(55.3 \%)$ & 0.001 \\
\hline Adherence to smoking, $\mathrm{n}(\%)$ & $6(17.1 \%)$ & $16(34.0 \%)$ & 0.001 \\
\hline Framingham risk score & $2.55 \pm 1.05$ & $8.09 \pm 2.12$ & 0.001 \\
\hline Systolic BP, mm Hg & $122 \pm 5$ & $137 \pm 4$ & 0.001 \\
\hline Diastolic BP, mm Hg & $72 \pm 4$ & $87 \pm 5$ & 0.001 \\
\hline Heart rate, beats per $1 \mathrm{~min}$. & $66 \pm 6$ & $71 \pm 6$ & 0.01 \\
\hline $\mathrm{GFR}, \mathrm{mL} / \mathrm{min} / 1.73 \mathrm{~m}^{2}$ & $\begin{array}{c}102.1(91.4- \\
113.2)\end{array}$ & $92.5(83.1-107.4)$ & 0.12 \\
\hline $\mathrm{HbA} 1 \mathrm{c}, \%$ & $4.75(4.36-5.12)$ & $6.82(4.61-5.37)$ & 0.001 \\
\hline $\begin{array}{l}\text { Fasting blood glucose, } \\
\mathrm{mmol} / \mathrm{L}\end{array}$ & $4.52(4.43-4.76)$ & $5.46(4.23-4.76)$ & 0.01 \\
\hline Insulin, $\mu \mathrm{U} / \mathrm{mL}$ & $4.98(1.5-14.1)$ & $14.2(12.5-15.7)$ & 0.001 \\
\hline HOMA-IR, mmol/L $\times \mu \mathrm{U} / \mathrm{mL}$ & $1.01(0.91-1.07)$ & $3.45(3.22-3.78)$ & 0.001 \\
\hline Creatinine, $\mu \mathrm{mol} / \mathrm{L}$ & $62.1(55.7-82.4)$ & $72.3(56.1-86.9)$ & 0.24 \\
\hline Total cholesterol, mmol/L & $4.76(4.21-5.05)$ & $5.3(4.5-5.9)$ & 0.001 \\
\hline LDL-C, mmol/L & $3.10(2.78-3.21)$ & $3.48(3.30-4.07)$ & 0.001 \\
\hline $\mathrm{HDL}-\mathrm{C}, \mathrm{mmol} / \mathrm{L}$ & $1.13(1.05-1.17)$ & $1.01(0.90-1.13)$ & 0.001 \\
\hline $\mathrm{TG}, \mathrm{mmol} / \mathrm{L}$ & $1,18(1.07-1.30)$ & $1.77(1.62-1.95)$ & 0.001 \\
\hline hs-CRP, mg/L & $4.11(0.97-5.03)$ & $7.87(4.92-9.43)$ & 0.001 \\
\hline sRANKL, pg/mL & $16.10(2.1-30.1)$ & $24.10(14.7-36.9)$ & 0.002 \\
\hline Osteoprotegerin, pg/mL & $88.3(37.5-136.6)$ & $718.5(572.1-846.2)$ & 0.001 \\
\hline Adiponectin, mg/L & $6.17(3.44-10.15)$ & $13.61(9.74-22.35)$ & 0.001 \\
\hline $25(\mathrm{OH}) \mathrm{D} 3, \mathrm{nmol} / \mathrm{L}$ & $98(89-112)$ & $67(16-126)$ & 0.001 \\
\hline 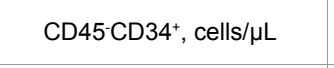 & $\begin{array}{l}0.092(0.076- \\
0.108)\end{array}$ & $0.060(0.055-0.066)$ & 0.001 \\
\hline CD14+CD309 ${ }^{+}$, cells $/ \mu \mathrm{L}$ & $\begin{array}{l}0.426(0.370- \\
0.574)\end{array}$ & $0.335(0.257-0.418)$ & 0.001 \\
\hline 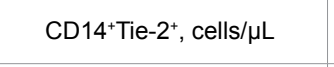 & $\begin{array}{l}0.045(0.023- \\
0.069)\end{array}$ & $0.031(0.021-0.040)$ & 0.001 \\
\hline CD309+ Tie-2 ${ }^{+}$, cells $/ \mu \mathrm{L}$ & $\begin{array}{l}0.038(0.026- \\
0.051)\end{array}$ & $0.022(0.017-0.032)$ & 0.001 \\
\hline $\mathrm{CD} 14^{+} \mathrm{CD} 309^{+} \mathrm{Tie}^{-2^{+}}$, cells $/ \mu \mathrm{L}$ & $\begin{array}{l}0.047(0.025- \\
0.071)\end{array}$ & $0.032(0.025-0.040)$ & 0.001 \\
\hline
\end{tabular}

Note: Data are presented as mean and \pm SE; median and $25-75 \%$ IQR. Categorical variables are expressed as numerous ( $n$ ) and percentages (\%). Statistical comparisons are made using Mann-Whitney test with significance levels of $<0.05$ (for 2-tailed). IQR: Inter Quartile Range; BMI: Body Mass Index; TG: Triglycerides; BP: Blood Pressure; GFR: Glomerular Filtration Rate; HDL-C: High-Density Lipoprotein Cholesterol; LDL-C: Low-Density Lipoprotein Cholesterol; hs-CRP: High Sensitive C Reactive Protein; sRANKL: Serum Receptor Activator of NF-KB ligand; HbA1c: Glycated Hemoglobin.

Table 1: General characteristics of patients participating in the study. 
Citation: Berezin AE, Kremzer AA, Martovitskaya YV, Berezina TA (2016) Non-Classical Progenitor Mononuclears in Metabolic Syndrome: The Role of Serum 25-Hydroxyvitamin D3. Clin Med Biochem 2: 115. doi:10.4172/2471-2663.1000115
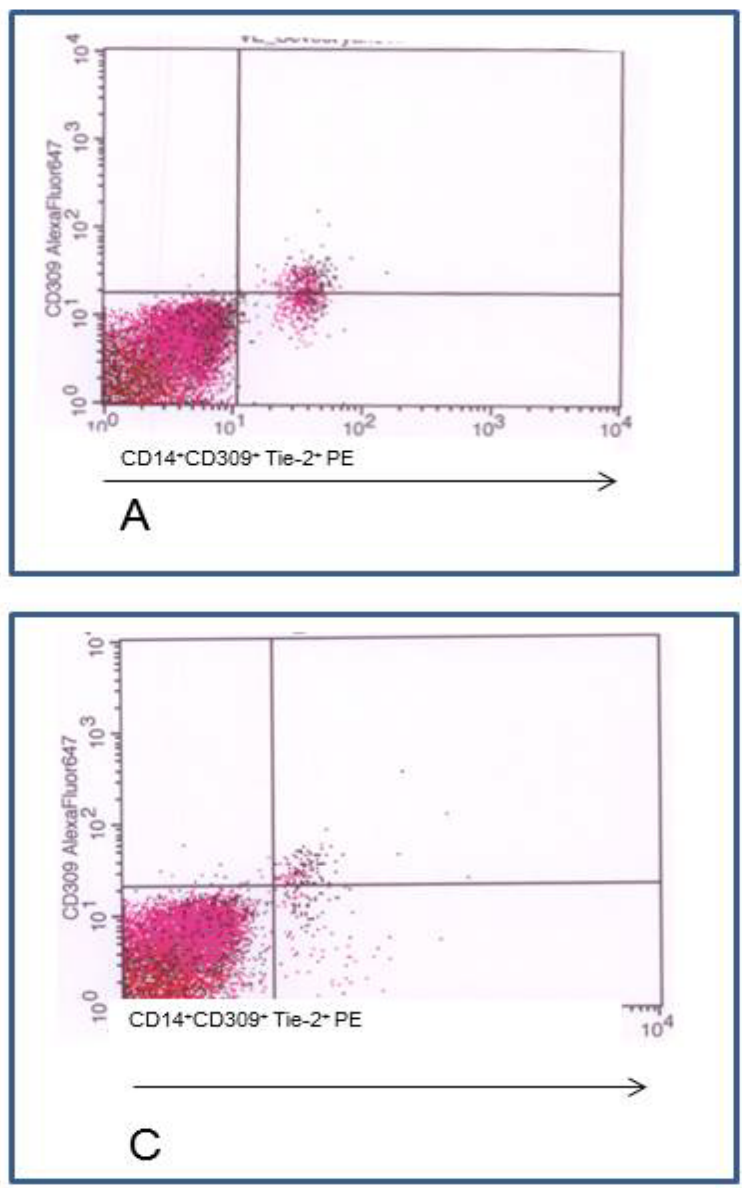
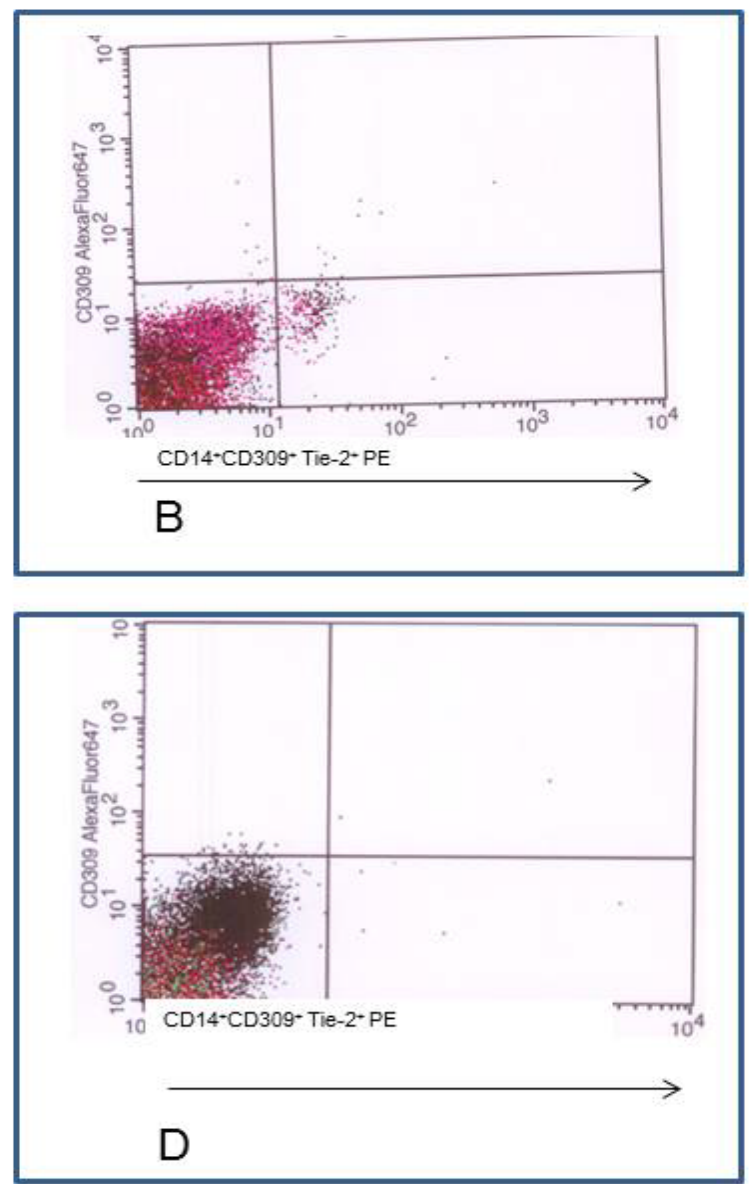

Figure 1: The sample of flow cytometry picture represented $\mathrm{CD} 14^{+} \mathrm{CD} 309+$ anti-Tie2 ${ }^{+}$cells in MetS patients with plasma level of $25(\mathrm{OH}) \mathrm{D} 3>100 \mathrm{nmol} / \mathrm{L}(\mathrm{Figure}$ $1 \mathrm{~A}$ ), ranged from 50 to $100 \mathrm{nmol} / \mathrm{L}$ (Figure $1 \mathrm{~B}$ ), ranged from 30 to $50 \mathrm{nmol} / \mathrm{L}$ (Figure $1 \mathrm{C}$ ), and $<30 \mathrm{nmol} / \mathrm{L}$ (Figure 1D).

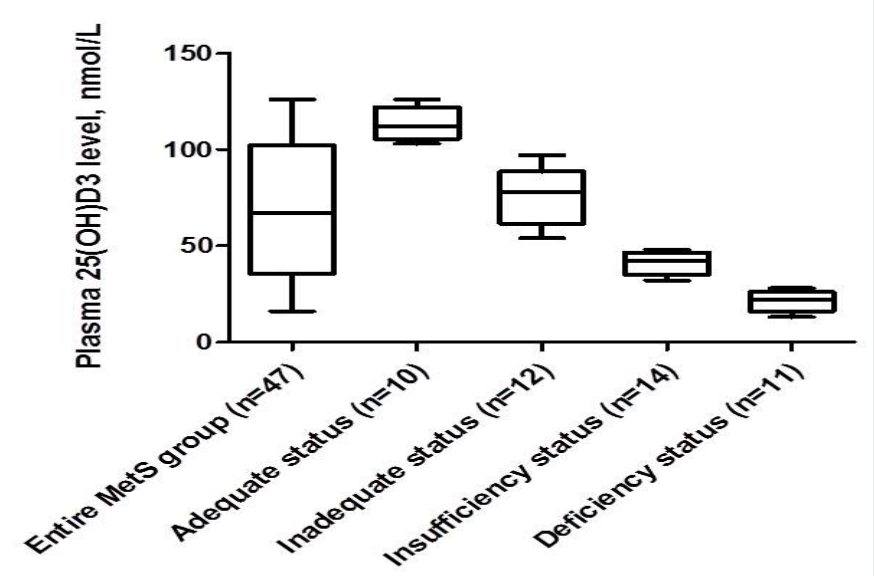

Vitamin $D$ status in Mets patients

Figure 2: The mean plasma levels of $25(\mathrm{OH}) \mathrm{D} 3$ in MetS cohorts. Values are reported as median and IQR, and were compared using ANOVA. The line within the box represents the median value; the top and bottom lines of the box reflect the $25^{\text {th }}$ and $75^{\text {th }}$ percentile respectively; the top and bottom vertical lines outside of the boxes represent $10^{\text {th }}$ and $90^{\text {th }}$ percentile respectively.
In multivariate linear regression analyses plasma level of $25(\mathrm{OH})$ D3 has associated with numerous of $\mathrm{CD} 14^{+} \mathrm{CD} 309^{+} \mathrm{Tie}-2^{+}$cells $(\mathrm{r}=0.406, \mathrm{P}=0.001)$, osteoprotegerin $(\mathrm{r}=-0.34, \mathrm{P}=0.001)$, body mass index $(\mathrm{r}=-0.41, \mathrm{P}=0.001), \mathrm{HbAlc}(\mathrm{r}=-0.33, \mathrm{P}=0.003)$, age $(\mathrm{r}=-0.35$, $\mathrm{P}=0.001)$, dyslipidemia $(\mathrm{r}=-0.31, \mathrm{P}=0.001)$, hs-CRP $(\mathrm{r}=-0.32, \mathrm{P}=0.001)$, triglycerides $(\mathrm{r}=-0.28, \quad \mathrm{P}=0.001)$, HOMA-IR $(\mathrm{r}=0.28, \mathrm{P}=0.003)$, HDL-C $(r=-0.27, P=0.001)$, and adiponectin $(r=-0.29, P=0.002)$. After adjustment on body mass index and age plasma level of 25(OH)D3 remained to be associated with numerous of $\mathrm{CD} 14^{+} \mathrm{CD} 309^{+} \mathrm{Tie}^{2} 2^{+}$cells $(\mathrm{r}=0.38, \mathrm{P}=0.001)$, osteoprotegerin $(\mathrm{r}=-0.34, \mathrm{P}=0.003)$, hs-CRP $(\mathrm{r}=-$ $0.31, \mathrm{P}=0.001)$ and adiponectin $(\mathrm{r}=-0.28, \mathrm{P}=0.002)$.

The log regression analysis have exhibited graduated effect of vitamin D status on depletion of numerous of $\mathrm{CD} 14^{+} \mathrm{CD} 309^{+} \mathrm{Tie}-2^{+}$cells (Table 3). In univariate model, decreased number of $\mathrm{CD} 14^{+} \mathrm{CD} 309^{+} \mathrm{Tie}-2^{+}$ cells has demonstrated a closely relation to deficiency or insufficiency vitamin D status (OR 1.16; $95 \%$ CI 1.05 to $1.23 ; \mathrm{P}=0.001$ and $\mathrm{OR}$ 1.06 ; $95 \%$ CI 1.01 to $1.12 ; \mathrm{P}=0.001$, respectively) rather than adequate vitamin D status (OR 1.02; 95\% CI 0.98 to $1.05 ; \mathrm{P}=0.46$ ). Therefore, osteoprotegerin, hs-CRP, adiponectin, and sRANKL have a significant impact of dependent variable. Interestingly, deficiency vitamin D status in multivariate log-regression model appeared to be remained an independent predictor to depletion of numerous of $\mathrm{CD} 14^{+} \mathrm{CD} 309^{+}$Tie$2^{+}$cells (OR 1.12; 95\% CI 1.06 to $1.19 ; \mathrm{P}=0.002$ ), whereas other vitamin $\mathrm{D}$ statuses were not found as predictors. Contrary, osteoprotegerin, hs- 
Citation: Berezin AE, Kremzer AA, Martovitskaya YV, Berezina TA (2016) Non-Classical Progenitor Mononuclears in Metabolic Syndrome: The Role of Serum 25-Hydroxyvitamin D3. Clin Med Biochem 2: 115. doi:10.4172/2471-2663.1000115

Page 5 of 8

\begin{tabular}{|c|c|c|c|c|c|}
\hline \multirow{2}{*}{ Variables } & \multicolumn{4}{|c|}{ Plasma level of $25(\mathrm{OH}) \mathrm{D} 3$} & \multirow{2}{*}{$P$ value } \\
\hline & $>100 \mathrm{nmol} / \mathrm{L}(\mathrm{n}=10)$ & 50 to $100 \mathrm{nmol} / \mathrm{L}(\mathrm{n}=12)$ & 30 to $50 \mathrm{nmol} / \mathrm{L}(\mathrm{n}=14)$ & $<30 \mathrm{nmol} / \mathrm{L}(\mathrm{n}=11)$ & \\
\hline Age, years & $46.40 \pm 1.88$ & $46.90 \pm 2.26$ & $47.10 \pm 3.00$ & $49.80 \pm 2.55$ & 0.046 \\
\hline Males, n (\%) & $6(60.0 \%)$ & $8(66.7 \%)$ & $9(64.2 \%)$ & $7(63.6 \%)$ & 0.74 \\
\hline $\mathrm{BMI}, \mathrm{kg} / \mathrm{m}^{2}$ & $27.1(16.2-29.6)$ & $28.5(17.1-30.3)$ & $28.9(17.5-31.8)$ & $29.5(19.9-32.1)$ & 0.22 \\
\hline Waist circumference, sm & $93(71-104)$ & $92(68-106)$ & $92(69-103)$ & $94(71-106)$ & 0.88 \\
\hline Hypertension, n (\%) & $7(70.0 \%)$ & $8(66.7 \%)$ & $10(71.4 \%)$ & $7(63.6 \%)$ & 0.78 \\
\hline Dyslipidemia, n (\%) & $4(40.0 \%)$ & $7(58.3 \%)$ & $9(62.3 \%)$ & $6(54.5 \%)$ & 0.044 \\
\hline Adherence to smoking, $\mathrm{n}(\%)$ & $4(40.0 \%)$ & $4(33.3 \%)$ & $5(35.7 \%)$ & $5(45.5 \%)$ & 0.42 \\
\hline Framingham risk score & $7.88 \pm 1.95$ & $7.96 \pm 2.03$ & $8.07 \pm 2.11$ & $8.12 \pm 1.80$ & 0.44 \\
\hline Systolic BP, mm Hg & $137 \pm 4$ & $135 \pm 4$ & $137 \pm 5$ & $136 \pm 5$ & 0.92 \\
\hline Diastolic BP, mm Hg & $88 \pm 5$ & $88 \pm 4$ & $87 \pm 7$ & $87 \pm 5$ & 0.92 \\
\hline Heart rate, beats per $1 \mathrm{~min}$. & $71 \pm 6$ & $70 \pm 5$ & $71 \pm 5$ & $72 \pm 5$ & 0.94 \\
\hline GFR, $\mathrm{mL} / \mathrm{min} / 1.73 \mathrm{~m}^{2}$ & $92.5(83.1-107.4)$ & $101.2(88.3-109.2)$ & $94.5(85.0-106.1)$ & $90.3(82.3-102.7)$ & 0.52 \\
\hline $\mathrm{HbA} 1 \mathrm{c}, \%$ & $6.75(4.58-5.30)$ & $6.79(4.60-5.34)$ & $6.83(4.57-5.35)$ & $6.87(4.72-5.41)$ & 0.038 \\
\hline Fasting blood glucose, $\mathrm{mmol} / \mathrm{L}$ & $5.44(4.24-4.72)$ & $5.45(4.21-4.77)$ & $5.46(4.26-4.80)$ & $5.47(4.25-4.71)$ & 0.22 \\
\hline Insulin, $\mu \mathrm{U} / \mathrm{mL}$ & $14.0(12.3-15.2)$ & $14.2(12.1-15.0)$ & $14.3(12.0-15.8)$ & $14.3(12.9-16.1)$ & 0.49 \\
\hline HOMA-IR, $\mathrm{mmol} / \mathrm{L} \times \mu \mathrm{U} / \mathrm{mL}$ & $3.38(3.19-3.72)$ & $3.41(3.20-3.71)$ & $3.47(3.20-3.75)$ & $3.48(3.29-3.80)$ & 0.042 \\
\hline Creatinine, $\mu \mathrm{mol} / \mathrm{L}$ & $72.3(56.1-86.9)$ & $70.5(53.7-83.2)$ & $72.9(56.0-86.5)$ & $74.1(59.2-91.4)$ & 0.66 \\
\hline Total cholesterol, mmol/L & $5.2(4.4-5.7)$ & $5.3(4.5-5.7)$ & $5.3(4.6-6.0)$ & $5.4(4.5-6.1)$ & 0.72 \\
\hline LDL-C, mmol/L & $3.45(3.29-4.04)$ & $3.46(3.30-4.06)$ & $3.48(3.28-4.10)$ & $3.48(3.29-4.08)$ & 0.62 \\
\hline $\mathrm{HDL}-\mathrm{C}, \mathrm{mmol} / \mathrm{L}$ & $1.09(1.01-1.15)$ & $1.06(0.92-1.12)$ & $1.01(0.90-1.10)$ & $1.01(0.88-1.06)$ & 0.036 \\
\hline $\mathrm{TG}, \mathrm{mmol} / \mathrm{L}$ & $1.47(1.32-1.63)$ & $1.56(1.44-1.68)$ & $1.78(1.50-2.13)$ & $1.99(1.53-2.19)$ & 0.044 \\
\hline hs-CRP, mg / L & $7.65(4.90-9.15)$ & $7.80(5.03-9.44)$ & $7.88(4.99-9.73)$ & $7.90(5.11-10.25)$ & 0.22 \\
\hline sRANKL, pg / mL & $19.70(13.82-30.10)$ & $21.40(15.1-32.7)$ & $22.90(15.3-33.4)$ & $27.60(15.90-37.15)$ & 0.18 \\
\hline Osteoprotegerin, $\mathrm{pg} / \mathrm{mL}$ & $693.2(551.1-811.6)$ & $703.9(565.1-822.6)$ & $715.2(580.2-850.1)$ & $727.6(583.5-870.1)$ & 0.028 \\
\hline Adiponectin, $\mathrm{mg} / \mathrm{L}$ & $11.55(8.87-14.92)$ & $12.87(9.15-16.62)$ & $13.25(9.80-17.75)$ & $17.90(9.97-22.76)$ & 0.018 \\
\hline CD45-CD34+, cells $/ \mu \mathrm{L}$ & $0.062(0.056-0.068)$ & $0.061(0.054-0.068)$ & $0.060(0.053-0.066)$ & $0.057(0.052-0.064)$ & 0.06 \\
\hline $\mathrm{CD} 14^{+} \mathrm{CD}^{2} 09^{+}$, cells $/ \mu \mathrm{L}$ & $0.359(0.270-0.425)$ & $0.341(0.263-0.416)$ & $0.335(0.234-0.402)$ & $0.329(0.257-0.418)$ & 0.10 \\
\hline 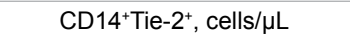 & $0.036(0.028-0.044)$ & $0.033(0.024-0.042)$ & $0.028(0.019-0.038)$ & $0.028(0.019-0.036)$ & 0.07 \\
\hline 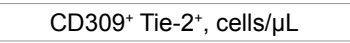 & $0.027(0.021-0.032)$ & $0.025(0.019-0.030)$ & $0.022(0.016-0.028)$ & $0.021(0.014-0.027)$ & 0.12 \\
\hline CD14+CD309+ Tie-2+, cells $/ \mu \mathrm{L}$ & $0.039(0.032-0.047)$ & $0.035(0.028-0.044)$ & $0.030(0.022-0.041)$ & $0.028(0.016-0.033)$ & 0.026 \\
\hline
\end{tabular}

Note: Data are presented as mean and $\pm \mathrm{SE}$ or $95 \% \mathrm{Cl}$; median and $25-75 \%$ IQR. Categorical variables are expressed as numerous ( $\mathrm{n}$ ) and percentages (\%). $\mathrm{P}$-value is a comparison of mean or median variables between both cohorts (ANOVA test).

Table 2: Demographic, risk factors, blood pressure, circulating biomarkers, and endothelial-derived microparticles in MetS and patients.

\begin{tabular}{|c|c|c|c|c|c|c|}
\hline \multirow{3}{*}{ Variables } & \multicolumn{6}{|c|}{ Dependent variable: numerous of CD14+CD309+ ${ }^{+}$Tie- $2^{+}$cells } \\
\hline & \multicolumn{3}{|c|}{ Univariate log-regression } & \multicolumn{3}{|c|}{ Multivariate log-regression } \\
\hline & OR & $95 \% \mathrm{Cl}$ & $P$ value & OR & $95 \% \mathrm{Cl}$ & $P$ value \\
\hline $25(\mathrm{OH}) \mathrm{D} 3<30 \mathrm{nmol} / \mathrm{L}$ vs $\geq 30 \mathrm{nmol} / \mathrm{L}$ & 1.16 & $1.05-1.23$ & 0.001 & 1.12 & $1.06-1.19$ & 0.002 \\
\hline $25(\mathrm{OH}) \mathrm{D} 3<50 \mathrm{nmol} / \mathrm{L}$ vs $\geq 50 \mathrm{nmol} / \mathrm{L}$ & 1.06 & $1.01-1.12$ & 0.001 & 1.04 & $0.99-1.07$ & 0.10 \\
\hline $25(\mathrm{OH}) \mathrm{D} 3<100 \mathrm{nmol} / \mathrm{L}$ vs $\geq 100 \mathrm{nmol} / \mathrm{L}$ & 1.02 & $0.98-1.05$ & 0.46 & \multicolumn{3}{|c|}{-} \\
\hline OPG per $225 \mathrm{pg} / \mathrm{mL}$ & 1.12 & $1.06-1.19$ & 0.001 & 1.10 & $1.04-1.15$ & 0.003 \\
\hline hs-CRP per $2.5 \mathrm{mg} / \mathrm{L}$ & 1.08 & $1.05-1.13$ & 0.003 & 1.05 & $1.02-1.09$ & 0.001 \\
\hline Adiponectin per $1.5 \mathrm{mg} / \mathrm{L}$ & 1.05 & $1.02-1.09$ & 0.001 & 1.03 & $1.01-1.06$ & 0.046 \\
\hline sRANKL per $2.5 \mathrm{pg} / \mathrm{mL}$ & 1.04 & $1.02-1.07$ & 0.001 & 1.02 & $1.00-1.04$ & 0.054 \\
\hline
\end{tabular}

BMI: Body Mass Index; OPG: Osteoprotegerin; hs-CRP: High-Sensitive C-Reactive Protein; OR: Odds Ratio; Cl: Confidence Interval

Table 3: The impact of vitamin D status and inflammatory cytokines on numerous of CD $14^{+} \mathrm{CD} 309^{+}$Tie- $2^{+}$cells: The results of univariate and multivariate age- and BMIadjusted log-regression analysis.

CRP, adiponectin have exhibited an independent impact on depletion of numerous of $\mathrm{CD} 14^{+} \mathrm{CD} 309^{+}$Tie- $2^{+}$cells.

Using C-statistics for Models with plasma level of $25(\mathrm{OH})$ $\mathrm{D} 3<30 \mathrm{nmol} / \mathrm{L}$, and circulating biomarkers (osteoprotegerin, hs-CRP, and adiponectin) as Continuous Variables we found that adding of combination of inflammatory biomarkers (osteoprotegerin, hs-CRP, and adiponectin) to the based model (deficiency vitamin $\mathrm{D}$ status defined as plasma level of $25(\mathrm{OH}) \mathrm{D} 3<30 \mathrm{nmol} / \mathrm{L}$ ) improved the relative IDI by $7.2 \%$ for decreased numerous of CD $14^{+} \mathrm{CD} 309^{+}$Tie- $2^{+}$cells (Table 4 ).

When we used another model constructed on entering variables, three biomarkers (osteoprotegerin, hs-CRP, and adiponectin) avoid to improve significantly predictive model based on plasma level of $25(\mathrm{OH})$ $\mathrm{D} 3<30 \mathrm{nmol} / \mathrm{L}$ for decreased numerous of $\mathrm{CD} 14^{+} \mathrm{CD} 309^{+} \mathrm{Tie}-2^{+}$ cells (Table 5). In patient study population for category-free NRI, $3 \%$ of events $(\mathrm{p}=0.16)$ and $4 \%$ of non-events $(\mathrm{p}=0.12)$ were correctly reclassified by the addition of circulating inflammatory biomarkers (osteoprotegerin, hs-CRP, and adiponectin) to the base model for decreased numerous of $\mathrm{CD} 14^{+} \mathrm{CD} 309^{+}$Tie- $2^{+}$cells.

\section{Discussion}

The results of the study exhibited that vitamin D deficiency status was found statistically significant predictor for decreased proangiogenic 


\begin{tabular}{|c|c|c|c|c|}
\hline \multirow{2}{*}{ Models } & \multicolumn{4}{|c|}{ Dependent variable: numerous of $\mathrm{CD} 14^{+} \mathrm{CD} 309^{+} \mathrm{Tie}-2^{+}$cells } \\
\hline & AUC $(95 \% \mathrm{Cl})$ & $\triangle \mathrm{AUC}$ & IDI ( \pm SE) & Relative IDI (\%) \\
\hline Model 1 (based model: plasma level of $25(\mathrm{OH}) \mathrm{D} 3<30 \mathrm{nmol} / \mathrm{L}$ ) & 0.676 & - & - & - \\
\hline Model 1 + OPG & 0.690 & - & - & - \\
\hline Model 1+OPG vs Model 1 & - & $0.014 ; P=0.46$ & $0.02 \pm 0.010$ & $2.2 \%$ \\
\hline Model 1+hs-CRP & 0.685 & - & - & - \\
\hline Model 1+hs-CRP vs Model 1 & - & $0.009 ; P=0.74$ & $0.01 \pm 0.008$ & $1.7 \%$ \\
\hline Model 1+OPG + hs-CRP & 0.689 & - & - & - \\
\hline Model 1+OPG+hs-CRP vs Model 1 & - & $0.013 ; P=0.48$ & $0.02 \pm 0.009$ & $1.9 \%$ \\
\hline Model 1+adiponectin & 0.682 & - & - & - \\
\hline Model 1 +adiponectin vs Model 1 & - & $0.006 ; P=0.78$ & $0.01 \pm 0.006$ & $1.6 \%$ \\
\hline Model 1+adiponectin+OPG & 0.694 & - & - & - \\
\hline Model $1+$ adiponectin+OPG vs Model 1 & - & $0.018 ; P=0.12$ & $0.02 \pm 0.008$ & $2.9 \%$ \\
\hline Model 1+hs-CRP+OPG+adiponectin & 0.720 & - & - & - \\
\hline Model 1+ hs-CRP+OPG+adiponectin vs Model 1 & - & $0.044 ; P<0.05$ & $0.03 \pm 0.011$ & $7.2 \%$ \\
\hline
\end{tabular}

Note: Relative IDI - calculated as the ratio of IDI over the discrimination slope of the model without plasma level of $25(\mathrm{OH}) \mathrm{D} 3<30 \mathrm{nmol} / \mathrm{L}$. AUC: Area Under Curve, SE: Standard Error, OPG: Osteoprotegerin, hs-CRP: High Sensitive C-Reactive Protein

Table 4: C-statistics for Models with plasma level of $25(\mathrm{OH}) \mathrm{D} 3<30 \mathrm{nmol} / \mathrm{L}$, hs-CRP, OPG, and adiponectin as Continuous Variables

\begin{tabular}{|c|c|}
\hline Variables & Model 2 vs Model 1 \\
\hline Categorical NRI & $0.11(95 \% \mathrm{Cl}=0.09-0.13)$ \\
\hline $\begin{array}{c}\text { Percentage of events correctly } \\
\text { reclassified }\end{array}$ & $2 \%(p=0.14)$ \\
\hline $\begin{array}{c}\text { Percentage of non-events correctly } \\
\text { reclassified }\end{array}$ & $3 \%(p=0.12)$ \\
\hline $\begin{array}{c}\text { Categorical free NRI } \\
\text { Percentage of events correctly } \\
\text { reclassified }\end{array}$ & $0.13(95 \% \mathrm{Cl}=0.10-0.15)$ \\
\hline $\begin{array}{c}\text { Percentage of non-events correctly } \\
\text { reclassified }\end{array}$ & $3 \%(p=0.16)$ \\
\hline
\end{tabular}

Note: Model 1, plasma level of $25(\mathrm{OH}) \mathrm{D} 3<30 \mathrm{nmol} / \mathrm{L}$; Model 2, plasma level of 25(OH)D3<30 nmol/L+hs-CRP+OPG+adiponectin

Table 5: Prediction Performance Analyses for Models with plasma level of $25(\mathrm{OH})$ $\mathrm{D} 3<30 \mathrm{nmol} / \mathrm{L}$ and inflammatory biomarkers (hs-CRP, OPG and adiponectin) as Continuous Variables for decreased numerous of $\mathrm{CD}_{14}{ }^{+} \mathrm{CD} 309^{+} \mathrm{Tie}-2^{+}$cells in MetS individuals.

progenitor mononuclears labelled as $\mathrm{CD} 14^{+} \mathrm{CD} 309^{+} \mathrm{Tie}-2^{+}$cells in MetS patients without known CV disease. Recently the depletion of proangiogenic subsets of circulating progenitor mononuclears bonemarrow originating received from peripheral blood was found as a marker of asymptomatic atherosclerosis and CV events/disease [28-30]. There is large body of evidence regarding closely inversely association between serum level of inflammatory biomarkers (osteoprotegerin, hs$\mathrm{CRP}$, and adiponectin) and numbers/functionality of proangiogenic progenitor mononuclears in dysmetabolic patients with known $\mathrm{CV}$ disease [31]. The exaggerated oxidative stress via osteoprotegerin, hs-CRP, and adiponectin may enhance epigenetic mechanisms affected methylation-related alteration of gene promoter region and leads to damage of DNA in target cells in diabetes [31,32]. These pathways remain their relevance as triggers of impaired function of circulating angiogenic progenitor mononuclear cells [33,34]. They are considered as one of clue in forming metabolic memory, which mediates manifestation and progression of $\mathrm{CV}$ disease in diabetes [35]. Moreover, $25(\mathrm{OH}) \mathrm{D} 3$ may regulate a functionality of circulating angiogenic progenitor mononuclear cells and via epigenetic pathways attenuate their reparative capacity $[36,37]$. Taken together deficiency of $25(\mathrm{OH}) \mathrm{D} 3$ might be a clue coordinating altered repair ability of circulating progenitor mononuclears and inflammatory response in MetS.

We have been speculated that $25(\mathrm{OH}) \mathrm{D} 3$ could be involved in pathogenesis of MetS and CV complications through molecular mechanisms of controlling the proliferation, differentiation, and function of mononuclear progenitor cells contributing endothelium reparation.
The results of our study have exhibited that MetS beyond known $\mathrm{CV}$ disease and type 2 diabetes mellitus exerts depleted circulating angiogenic progenitor mononuclear cells compared with healthy volunteers. We also noticed that vitamin D status has closely associated not only with age, body mass index, dyslipidemia, HbA1c and HOMAIR, as well as serum level of inflammatory biomarkers and changes in numerous of subsets of progenitor mononuclears labelled CD14 ${ }^{+}$ $\mathrm{CD} 309^{+} \mathrm{Tie}^{-2}{ }^{+}$cells. Interestingly, after adjustment on body mass index and age plasma level of $25(\mathrm{OH}) \mathrm{D} 3$ remained to be related to numerous of angiogenic progenitor mononuclears and inflammatory biomarkers. Although osteoprotegerin, hs-CRP, adiponectin have effected depletion of numerous of $\mathrm{CD}^{+} 4^{+} \mathrm{CD} 309^{+} \mathrm{Tie}^{2+}$ cells, deficiency of $25(\mathrm{OH})$ D3 was found as an independent powerful predictor of decreased angiogenic progenitor mononuclears. Thus, we suggested being link between vitamin $\mathrm{D}$ status and impaired functionality of endogenous endothelial repair system that might be direct mechanism contributing vascular complications in MetS individuals.

Recent studies have shown a pivotal role of $25(\mathrm{OH}) \mathrm{D} 3$ in the formation of capillary-like structures, which are considered a certain step in angiogenesis de novo [38,39]. Because circulating mononuclear progenitor cells participate in maintaining endothelial integrity and vascular homeostasis, $25(\mathrm{OH}) \mathrm{D} 3$ deficiency may contribute to vascular dysfunction in MetS through altered differentiation of mononuclear progenitors into mature endothelial cells [40]. As one see from the results of our study, deficiency of $25(\mathrm{OH}) \mathrm{D} 3$ may be ascribed to impaired progenitor mononuclears' production in MetS via enhancing development of IR and low-grading inflammation.

We cannot exclude that epigenetic effect of glucose toxicity might contribute in manifestation of IR in mononuclear progenitor cells [41]. In this context, a negative impact of $25(\mathrm{OH}) \mathrm{D} 3$ as a potent inhibitor of neovascularization and cell migration on pattern of angiogenic progenitor mononuclears could be realize in case, when up-regulated inflammatory cascade cytokines are presented. Indeed, it is so difficult to explain why deficiency of 25(OH)D3 that is suitable for both metabolically healthy obesity and MetS may lead to controversial effects among these settings. It has been found that most metabolically healthy obese individuals have not exhibited highly risk of diabetes development, while IR has been presented [42]. Moreover, numerous of angiogenic progenitor mononuclears in this patients' population appears to be increased [43]. Contrary, MetS and type 3 diabetes mellitus individuals have demonstrated a decreased 
level of circulating progenitor mononuclears with angiogenic immune phenotypes [44], which independently predict CV and atherosclerotic disease progression [45,46]. It might be suggested that in MetS subjects vitamin $\mathrm{D}$ deficiency leading to an exacerbation of inflammation associates with $\mathrm{CV}$ disease development and processes via involving alteration of endothelium repair system, such as angiogenic progenitor mononuclear cells. Whether is causative relation between vitamin D deficiency and depleted number circulating progenitor mononuclears with angiogenic immune phenotypes is question that is addressed to the future investigations.

In conclusion, vitamin D status especially low level of $25(\mathrm{OH}) \mathrm{D} 3$ may associate with depletion of circulating number of proangiogenic progenitor mononuclear cells in MetS patients. More investigations are required to explain the link between vitamin D status and number circulating angiopoetic progenitor mononuclears in MetS patients without known CV disease.

\section{Study Limitations}

This study has some limitations. Due to small size of study population we cannot exclude possibility that other immune phenotypes of progenitor mononuclear cells might be affected vitamin D status. Therefore, the predictive role of age and body mass index requires to be elucidated carefully. However, the authors suppose that these restrictions might have no significant impact on the study data interpretation.

\section{Acknowledgements}

We thank all patients for their participation in the investigation, staff of the Regional Zaporozhye Hospital (Ukraine), and the doctors, nurses, and administrative staff in Regional Center of cardiovascular diseases (Zaporozhye Ukraine) and City Hospital \# 6 (Zaporozhye, Ukraine), general practices, and sitemanaged organizations that assisted with the study.

\section{References}

1. Alberti KG, Eckel RH, Grundy SM, Zimmet PZ, Cleeman JI, et al. (2009) Harmonizing the metabolic syndrome: a joint interim statement of the International Diabetes Federation Task Force on Epidemiology and Prevention; National Heart, Lung, and Blood Institute; American Heart Association; World Heart Federation; International Atherosclerosis Society; and International Association for the Study of Obesity. Circulation 120: 1640-1645.

2. Gami AS, Witt BJ, Howard DE, Erwin PJ, Gami LA, et al. (2007) Metabolic syndrome and risk of incident cardiovascular events and death: a systematic review and meta-analysis of longitudinal studies. J Am Coll Cardiol 49: 403-414.

3. Bayturan O, Tuzcu EM, Lavoie A, Hu T, Wolski K, et al. (2010) The metabolic syndrome, its component risk factors, and progression of coronary atherosclerosis. Arch Intern Med 170: 478-484

4. Strange RC, Shipman KE, Ramachandran S (2015) Metabolic syndrome: A review of the role of vitamin $D$ in mediating susceptibility and outcome. World J Diabetes 6: 896-911.

5. Parker J, Hashmi O, Dutton D, Mavrodaris A, Stranges S, et al. (2010) Levels of vitamin $D$ and cardiometabolic disorders: systematic review and meta-analysis. Maturitas 65: 225-236.

6. Amirbaigloo A, Hosseinpanah F, Sarvghadi F, Tohidi M, Eskandary PS, (2013) Absence of association between vitamin $D$ deficiency and incident metabolic syndrome: Tehran Lipid and Glucose Study. Metab Syndr Relat Disord 11: 236-42.

7. Chiu KC, Chu A, Go VL, Saad MF (2004) Hypovitaminosis D is associated with insulin resistance and beta cell dysfunction. Am J Clin Nutr 79: 820-825.

8. Miñambres I, de Leiva A, Pérez A (2014) Hypovitaminosis D and metabolic syndrome. Med Clin (Barc) 143: 542-547.

9. Nimitphong $\mathrm{H}$, Chanprasertyothin $\mathrm{S}$, Jongjaroenprasert $\mathrm{W}$, Ongphiphadhanakul B (2009) The association between vitamin D status and circulating adiponectin independent of adiposity in subjects with abnormal glucose tolerance. Endocrine 36: 205-210.
10. McGill AT, Stewart JM, Lithander FE, Strik CM, Poppitt SD (2008) Relationships of low serum vitamin D3 with anthropometry and markers of the metabolic syndrome and diabetes in overweight and obesity. Nutr J 7: 4.

11. Jablonski KL, Chonchol M, Pierce GL, Walker AE, Seals DR (2011) 25 -Hydroxyvitamin $D$ deficiency is associated with inflammation-linked vascular endothelial dysfunction in middle-aged and older adults. Hypertension 57: 63-69.

12. Sato M, Hiragun A (1988) Demonstration of 1 alpha,25-dihydroxyvitamin D3 receptor-like molecule in ST 13 and $3 \mathrm{~T} 3 \mathrm{~L} 1$ preadipocytes and its inhibitory effects on preadipocyte differentiation. J Cell Physiol 135: 545-550.

13. Bikle D (2009) Nonclassic actions of vitamin D. J Clin Endocrinol Metab 94 26-34.

14. Berezin AE (2016) Metabolic memory phenomenon in diabetes mellitus: achieving and perspectives. Diabetes \& Metabolic Syndrome: Clinical Research \& Reviews.

15. Berezin AE, Kremzer AA, Berezina TA, Yu MV (2015) The impact of lowgrading inflammation on circulating endothelial-derived progenitor cells in patients with metabolic syndrome and diabetes mellitus. J Endocrinology and Diabetes 2: 8-16.

16. Kuliszewski MA, Ward MR, Kowalewski JW, Smith AH, Stewart DJ, et al. (2013) A direct comparison of endothelial progenitor cell dysfunction in rat metabolic syndrome and diabetes. Atherosclerosis 226: 58-66.

17. Kotlinowski J, Dulak J, Józkowicz A (2013) Type 2 diabetes mellitus impairs endothelial progenitor cells functions. Postepy Biochem 59: 257-266.

18. Lombardo MF, lacopino P, Cuzzola M, Spiniello E, Garreffa C, et al. (2012) Type 2 diabetes mellitus impairs the maturation of endothelial progenitor cells and increases the number of circulating endothelial cells in peripheral blood. Cytometry A 81: 856-864.

19. Yiu YF, Chan YH, Yiu KH, Siu CW, Li SW, et al. (2011) Vitamin D deficiency is associated with depletion of circulating endothelial progenitor cells and endothelial dysfunction in patients with type 2 diabetes. $\mathrm{J}$ Clin Endocrinol Metab 96: E830-835.

20. Gui J, Rohrbach A, Borns K, Hillemanns P, Feng L, et al. (2015) Vitamin D rescues dysfunction of fetal endothelial colony forming cells from individuals with gestational diabetes. Placenta 36: 410-418.

21. National Cholesterol Education Program (NCEP) Expert Panel on Detection Evaluation, and Treatment of High Blood Cholesterol in Adults (Adult Treatment Panel III) (2002) Third Report of the National Cholesterol Education Program (NCEP) Expert Panel on Detection, Evaluation, and Treatment of High Blood Cholesterol in Adults (Adult Treatment Panel III) final report. Circulation 106: 3143-3421.

22. Boyle CA, Dobson AJ, Egger G, Bennett SA (1993) Waist-to-hip ratios in Australia: a different picture of obesity. Aust J Nutr Diet 50: 57-64.

23. Levey AS, Stevens LA, Schmid CH, Zhang YL, Castro AF 3rd, et al (2009) A New Equation to Estimate Glomerular Filtration Rate. Ann Intern Med 150: 604-612.

24. Matthews DR, Hosker JP, Rudenski AS, Naylor BA, Treacher DF, et al. (1985) Homeostasis model assessment: insulin resistance and beta-cell function from fasting plasma glucose and insulin concentrations in man. Diabetologia 28: 412-419.

25. Tam CS, Xie W, Johnson WD, Cefalu WT, Redman LM, et al. (2012) Defining insulin resistance from hyperinsulinemic-euglycemic clamps. Diabetes Care 35: 1605-1610.

26. Tung JW, Parks DR, Moore WA, Herzenberg LA, Herzenberg LA (2004) New approaches to fluorescence compensation and visualization of FACS data. Clin Immunol 110: 277-283.

27. Hoffman RA (2005) Standardization, calibration, and control in flow cytometry Curr Protoc Cytom Chapter 1: Unit 1.

28. Berezin AE, Kremzer AA. (2014) Relationship between circulating endothelia progenitor cells and insulin resistance in none-diabetic patients with ischemic chronic heart failure. Diabetes \& Metabolic Syndrome: Clinical Research \& Reviews 8: 138-144.

29. Berezin AE, Kremzer AA (2013) Analysis of Various Subsets of Circulating Mononuclear Cells in Asymptomatic Coronary Artery Disease. J Clin Med 2: 32-44. 
Citation: Berezin AE, Kremzer AA, Martovitskaya YV, Berezina TA (2016) Non-Classical Progenitor Mononuclears in Metabolic Syndrome: The Role of Serum 25-Hydroxyvitamin D3. Clin Med Biochem Open Access 2: 115. doi:10.4172/2471-2663.1000115

30. Berezin AE (2015) Biological markers of cardiovascular diseases. Part 3: Diagnostic and prognostic value of biological markers in stratification of patients with cardiometabolic risk. Lambert Academic Publishing $\mathrm{GmbH}$, Moscow, $\mathrm{p}$ : 300

31. Mori K, Kitazawa R, Kondo T, Mori M, Hamada Y, et al. (2014) Diabetic osteopenia by decreased $\beta$-catenin signaling is partly induced by epigenetic derepression of SFRP-4 gene. PLoS One 9: e102797.

32. Hazra S, Jarajapu YP, Stepps V, Caballero S, Thinschmidt JS, et al. (2013) Long-term type 1 diabetes influences haematopoietic stem cells by reducing vascular repair potential and increasing inflammatory monocyte generation in a murine model. Diabetologia 56: 644-653.

33. Chakravarthy H, Beli E, Navitskaya S, O'Reilly S, Wang Q, et al. (2016) Imbalances in Mobilization and Activation of Pro-Inflammatory and Vascula Reparative Bone Marrow-Derived Cells in Diabetic Retinopathy. PLoS One 11: e0146829.

34. Barthelmes D, Irhimeh MR, Gillies MC, Karimipour M, Zhou M, et al. (2013) Diabetes impairs mobilization of mouse bone marrow-derived Lin(-)/ VEGF-R2(+) progenitor cells. Blood Cells Mol Dis 51: 163-173.

35. Wu H, Li R, Wei ZH, Zhang XL, Chen JZ, et al. (2016) Diabetes-Induced Oxidative Stress in Endothelial Progenitor Cells May Be Sustained by a Positive Feedback Loop Involving High Mobility Group Box-1. Oxidative Medicine and Cellular Longevity.

36. von Versen-Höynck F, Brodowski L, Dechend R, Myerski AC, Hubel CA (2014) Vitamin $D$ antagonizes negative effects of preeclampsia on fetal endothelial colony forming cell number and function. PLoS One 9: e98990.

37. Brodowski L, Burlakov J, Myerski AC, von Kaisenberg CS, Grundmann M, et al. (2014) Vitamin D prevents endothelial progenitor cell dysfunction induced by sera from women with preeclampsia or conditioned media from hypoxic placenta. PLoS One 9: e98527.
38. Grundmann M, Haidar M, Placzko S, Niendorf R, Darashchonak N, et al. (2012) Vitamin $D$ improves the angiogenic properties of endothelial progenitor cells. Am J Physiol Cell Physiol 303: C954-962.

39. Reynolds J, Ray D, Alexander MY, Bruce I (2015) Role of vitamin D in endothelial function and endothelial repair in clinically stable systemic lupus erythematosus. Lancet 385 Suppl 1: S83.

40. Saito H, Yamamoto Y, Yamamoto H (2012) Diabetes alters subsets of endothelial progenitor cells that reside in blood, bone marrow, and spleen. Am J Physiol Cell Physiol 302: C892-901.

41. Patel TP, Rawal K, Bagchi AK, Akolkar G, Bernardes N, et al. (2016) Insulin resistance: an additional risk factor in the pathogenesis of cardiovascular disease in type 2 diabetes. Heart Fail Rev 21: 11-23.

42. Singh H, Parthasarathy V, Farouk M, Venkatesan V (2016) Progenitor cells may aid successful islet compensation in metabolically healthy obese individuals. Med Hypotheses 86: 97-99.

43. Pires A, Martins P, Paiva A, Pereira AM, Marques M, et al. (2015) Circulating endothelial progenitor cells in obese children and adolescents. J Pediatr (Rio J) $91: 560-566$

44. Kang L, Chen Q, Wang L, Gao L, Meng K, Chen J, et al. (2009) Decreased mobilization of endothelial progenitor cells contributes to impaired neovascularization in diabetes. Clin Exp Pharmacol Physiol. 36: e47-56.

45. Schmidt-Lucke $C$, Rössig L, Fichtlscherer $S$, Vasa $M$, Britten $M$, et al. (2005) Reduced number of circulating endothelial progenitor cells predicts future cardiovascular events: proof of concept for the clinical importance of endogenous vascular repair. Circulation 111: 2981-2987.

46. Liao YF, Feng Y, Chen LL, Zeng TS, Yu F, et al (2014) Coronary heart disease risk equivalence in diabetes and arterial diseases characterized by endothelial function and endothelial progenitor cell. J Diabetes Complications 28: 214-218.
Citation: Berezin AE, Kremzer AA, Martovitskaya YV, Berezina TA (2016) Non-Classical Progenitor Mononuclears in Metabolic Syndrome: The Role of Serum 25-Hydroxyvitamin D3. Clin Med Biochem Open Access 2: 115 doi:10.4172/2471-2663.1000115 